\title{
Electromagnetic Radiation and Heat Transfer in Disperse Systems Consisting of Spherical and Cylindrical Particles
}

\author{
Lyudmila A.Uvarova, ${ }^{1, *}$, Irina $V$. Krivenko $^{2}$, Marina A. Smirnova ${ }^{3}$ and Alexey B. Nadykto ${ }^{1}$ \\ ${ }^{1}$ Moscow State Technological University "STANKIN", RU-127055, Moscow, Russia \\ ${ }^{2}$ Tver State Technical University, Department of General Physics, RU-170000, Tver, Russia \\ ${ }^{3}$ Tver State Technical University, Department of Computer Science and Applied Mathematics, RU-170026, Tver, Russia
}

\begin{abstract}
The article deals with the electromagnetic radiation transfer in systems of spherical disperse particles with different optical characteristics. A model of the electromagnetic radiation transfer in cylindrical particles containing a small volume of different chemical substance is developed. The substance differs substantially from that of the particle in a radiation absorption coefficient for the wavelength under study in the long wave approximation. The finite element method is used to calculate the temperature field for the system of spherical particles in a two-dimensional approximation. The configurations of particle packing is chosen on a random basis, which significantly complicated the calculations, the longitudinal and transverse diameters of particle clusters, the distance between centers of two largest particles, and similar natural geometric properties have been considered as characteristic system dimensions. The possibility of controlling heat transfer in such systems is studied. It follows from our model calculations that both electromagnetic and thermal interaction of dispersed particles can be noticeable at large distances between their centers; that near the boundary of the dispersed particle there is a thermal surface layer of the particle, where the temperature distribution is essentially heterogeneous. It is concluded that the thermal mechanism of destruction of a weakly absorbing particle due to a strong increase in temperature because of electromagnetic resonance in a neighboring particle with a strong absorption. It is established that the effect of collective influences in polydisperse system can change temperature by more than 1,5 times.
\end{abstract}

\section{Introduction}

Today, modeling the physical phenomena in disperse systems with various physicochemical properties is becoming increasingly important [1-4]. This is due to both the global environmental problem of atmospheric pollution when aerosols with their large total interface have a significant impact on all physicochemical processes in the global atmosphere, and modern technologies where dispersed substances are used [5]. In studying aerosols, it is important to take into account the nonlinear dependence of various characteristics of dispersed particles on temperature, electromagnetic field vectors and others. In the paper, influence of the electromagnetic radiation and heat transfer processes on dispersed systems exposed to intense electromagnetic radiation have been studied.

\section{Electromagnetic radiation transfer in systems of spherical disperseparticles}

Pair interactions of spherical disperse particles affected by monochromatic electromagnetic radiation are examined. The electromagnetic field energy absorbed by the particles and released in the unit volume of the $j$-th particle per unit time is a heat source $q_{j}$ initiated by radiation. It is determined as the squared absolute value of the electric-field vector amplitude at a given point [6]. The calculation method for electromagnetic field vector components was developed earlier on the basis of the Mie solution [5] and group representation theory [8]. To solve the problem, the authors localize the centers of the particles $\mathrm{O}_{1}$ and $\mathrm{O}_{2}$, the radii $\mathrm{R}_{1}$ and $\mathrm{R}_{2}$, and the directions of vectors $\boldsymbol{E}$ (external electromagnetic field strengths) and $\boldsymbol{k}$ (a wave vector) to the Cartesian axes (Figure 1) [9].

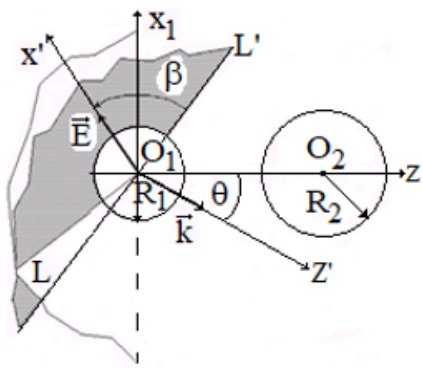

Fig. 1. System of two particles in the electromagnet radiation field.

The incident electric vector is harmonic timedependent and its amplitude can be written as follows: 


$$
\Delta E+k^{(j) 2} E=0
$$

where $k^{(j)}$ is a wave number in the substance $j$, $k^{2}=-k_{1} k_{2}, k_{1}=\frac{i \omega}{c}\left(\varepsilon+i \frac{4 \pi \sigma}{\omega}\right), k_{2}=i \frac{\omega}{c}, \omega$ is a cyclic frequency, $\varepsilon$ is medium permittivity, $\sigma$ is specific conductivity, $\mathrm{c}$ is velocity of light. The number $j$ takes the following values: $j=0$ (external environment), $j=1$ (particle No.1), $j=2$ (particle No.2). The same equation can be written for the magnetic vector.

The influence of the $k$-th particle can be considered if the field $\boldsymbol{E}_{\boldsymbol{j}}$ being external to $j$-th particle can be written as:

$$
E_{j}=E+E_{k}^{S}
$$

where $\boldsymbol{E}$ is an incident light vector, $\boldsymbol{E}_{\boldsymbol{k}}^{\boldsymbol{s}}$ isa wave scattered on a neighbor particle. The vectors $\boldsymbol{E}$ and $\boldsymbol{H}$ can be represented with electric $U$ and magnetic $V$ Debye potentials [5]. The Debye potentials of an incident field are described in the coordinate system $x^{\prime} y^{\prime} z$ ', the Debye potentials of the field scattered on the $k$-th particle are described in the coordinate system $x_{k} y_{k} z \quad(k=1,2)$. Further, the expressions obtained are represented as expansions of the eigen spherical harmonics of the $j$-th sphere. The Euler angles $\pi / 2-\beta, \theta, 3 \pi / 2$ completely define the rotation of $g$ about the point $\mathrm{O}_{1}$ transferring the coordinate system $x^{\prime} y^{\prime} z$ ' into $x_{1} y_{1} z$.

To calculate the heat source, the authors divide the particle into small volumes with practically constant $|\boldsymbol{E}|^{2}$. Practicing it we can examine volume average particles and calculate their heat source densities in respect to the intensity of the incident wave $q^{*}$ for different particle system alignments with the field vectors. Table 1 provides the example of such calculations for water aerosol particles with radii $R_{1}=R_{2}=1 \mu \mathrm{m}$. The distance between the particle centres is $R=20 \mu \mathrm{m}$. The length of the laser radiation wave initiating heat transfer is $\lambda=$ $10.6 \mu \mathrm{m}$. The ambient temperature is $T=273 \mathrm{~K}$. The coefficient of medium thermal conductivity is $\chi_{e}=24.1$ $\mathrm{mW} /(\mathrm{m} \cdot \mathrm{K})$, the coefficients of particle substance thermal conductivity are $\chi_{1}=\chi_{2}=566 \mathrm{~mW} / \mathrm{m} \cdot \mathrm{K}$. The complex refraction indices of particle substance (water) are1.173 $+\mathrm{i} \cdot 0.0823$. The medium refraction index is $n_{e}=1$.

Table 1. Dependence of $\mathrm{q}^{*}$ on Euler angles

\begin{tabular}{|c|c|c|}
\hline $\boldsymbol{\theta}$, degrees & $\boldsymbol{\beta}$,degrees & $\boldsymbol{q}_{i}{ }^{\boldsymbol{}} \cdot \mathbf{1 0}^{\mathbf{- 4}}, \mathbf{~ m}^{\mathbf{- 1}}$ \\
\hline 90 & 0 & 8.7 \\
\hline 90 & 90 & 5.7 \\
\hline 45 & 45 & 6.3 \\
\hline
\end{tabular}

The calculations show that the average density of heat sources significantly depends on the location of the system of two particles over the wave vector and the electric field intensity vector even at large distances between the particles.

In [10] has noted previously that not only electromagnetic interaction but also thermal one of disperse particles can be noticeable at large distances between their centers. The calculations show that the distribution is more heterogeneous near the boundary of the disperse particle than in the central regions, which allows us to speak about the existence of a thermal surface layer of a particle characterized by a highly inhomogeneous temperature field inside. The thermal interaction of two disperse particles with substantially different absorbing properties has been studied. The authors obtained the result indicating a thermal mechanism of low-absorbing particle destruction because of the strong temperature increase due to electromagnetic resonance in a neighbor particle with a high absorption [11].

\section{Electromagnetic radiation transfer in systems of cylindrical disperseparticles}

Another common geometric model of disperse particles is a cylinder or elongated spheroid. The method developed for calculating the amplitude square for this geometry [10] is appropriate under the condition that the radius of the cylinders is much smaller than the wavelength of the incident radiation. This restriction allows a bicylindrical coordinate system to be used, which greatly simplifies the solution and calculations $[12,13]$.

For these particle systems, $|\boldsymbol{E}|^{2} /\left|\boldsymbol{E}_{\mathbf{0}}\right|^{2}$ (the ratio of the squared absolute value of the light vector amplitude at the selected point inside the particle to the squared absolute value of the electric-field vector of the incident wave) is calculated. The ratio characterizes the heat source densities depending on the radius and angle coordinates in the cross-sections perpendicular to the cylinder axis.

Systems of inhomogeneous cylinders with different complex dielectric permittivities of the particle substance are examined. The calculations used the following values: $\lambda=0.2 \mathrm{~cm} ; \varepsilon_{1}=1$ (air); $\varepsilon_{2}=1.33-0.0009 i$ (ice).

Computational experiments have been carried out for two-layer cylinders with concentric (previously in [10]) and non-concentric layers (the inner region of the particle isa structure with a randomly distributed radius depending on the cylinder cross-section angle) of different chemical composition.

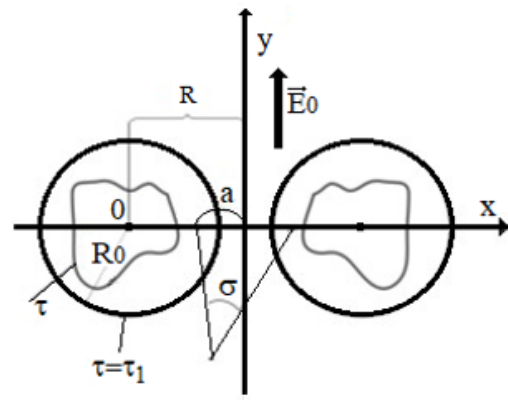

Fig. 2. System of cylinders with a nonconcentric layer.

Figure 2 shows a system of heterogeneous cylinders. Here $\tau, \sigma$ are curvilinear bicylindrical coordinates. The polar distance is denoted by $a$, the radius of a cylinder is $R_{0}$, the half of a distance between cylinders is $R$, the light vector of the incident plane monochromatic electromagnetic wave is $\boldsymbol{E}_{\mathbf{0}}$, and the cylinder coordinate 
surface in a bicylindrical coordinate system is $\tau_{1}$. The solution considered in this paper reduces to that given in [10], but here the inner boundary radius is reset for each selected point.

The distribution of $|\boldsymbol{E}|^{2} /\left|\boldsymbol{E}_{\mathbf{0}}\right|^{2}$ is found inside a cylindrical particle of radius $R_{0}=1 \mu \mathrm{m}$, with a circle radius of the selected section being $0.95 \mu \mathrm{m}$ with a random distribution of the inner boundary of the outer layer. In this case, the circle point can belong to both outer and inner layers. Complex permittivity for the inner layer is equal to ice; the values of the outer layer come to those from a model and correspond to forceful absorption.

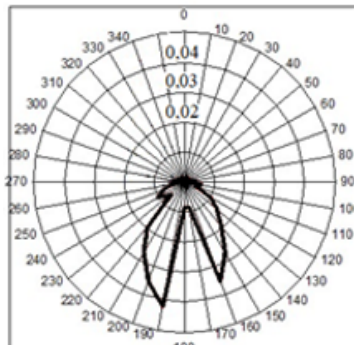

(a)

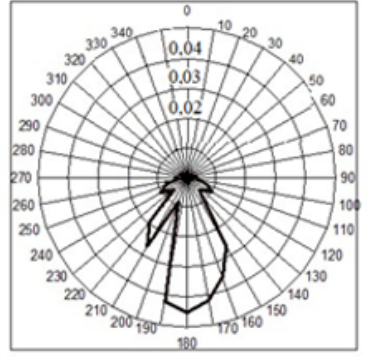

(b)
Fig.3. Distribution of $|\mathrm{E}|^{2} /\left|\mathrm{E}_{0}\right|^{2}$ over a cylinder cross-section.

Figures $3 a$ and $3 b$ correspond to different configurations of the outer layer inner boundary drawn at random. Resulting distributions depend on the outer layer thickness at a selected point and fit into the diagram calculated for the radius of the outer layer inner boundary corresponding to the minimum thickness of the outer layer.

\section{Heat transfer in disperse systems of various structures and configurations}

Heat transfer in dispersed systems of various composition, configuration, and particle sizes has been studied by the finite element method. Spherical particles of real sizes for natural aerosols are examined by models, with the collective effects being calculated and the dependence of system temperature on a particle size, etc. being estimated. The developed model program allows calculations for arbitrarily-shaped particles as well.

The model involves a rectangular area of $90 \times 70 \mu \mathrm{m}$ with spherical particles of radius 5 and $10 \mu \mathrm{m}$ placed in it. The number of particles range from one to nine; the particle packing in the area also varies. Particle numbering for mono- and polydisperse systems, as well as configurations of systems are given in [11].

Key findings of heat transfer in the dispersed system with compositionally homogeneous particles are published in [11]. Temperature field has been also calculated for the system containing particles of heterogeneous chemical composition. There have been attempts to analyze the influence of nonlinear temperature-source relationship on heat transfer in the dispersed system.

The authors consider two temperature-source relationships:
1. $q=q_{0}\left(\frac{T}{T_{\infty}}\right)^{\sigma}$ where parameter $\sigma$ ranges from 0.5 to 2 .

2. $q=q_{0} e^{\frac{E_{A}}{k T}}$ where parameter $\frac{E_{A}}{k T}$ varies within the range of $0.001-100$.

The main calculation data are given in [11]. With the nonlinear dependence of a heat source on temperature and an increase in number of particles, there occurs a significant system overheating. For example, if $\sigma=1$, the representative temperature in the configuration system containing eleven particles is nearly three times over that in the one-particle configuration.

From the analysis inspired by computational experiments we can conclude that particles of different sizes and thermophysical properties increase the temperature field non-uniformity in a system, with nonlinear effects being manifested under linear dependence of the source on temperature, whereas nonlinear dependence enhances these effects. If the system is polydisperse, presence of larger particles has a significant impact on such a distribution, and under boundary conditions of the third kind at the external boundary of the system, collective effects can change the temperature one-and-a-half times or even more. This influence is the greater, the more heterogeneous the system (in particle size and composition).

Based on a general approach to solving the problem of optimization of heat transfer in a system of $\mathrm{N}$ particles conditioned by heat sources in each particle, calculation formulas for determining optimal configuration parameters of the system with two spherical particles and optimal external effects are obtained. Representative data of numerical model calculations obtained from the formulas are given in $[14,15]$. To solve an optimization problem, the authors use the solutions of the heat transfer under electromagnetic radiation for two spherical particles $[9,10]$. Such governing parameters as external electromagnetic source power and configuration of particle packing have been used in calculations.

At the same time, a functional for the corresponding system with distributed parameters is minimized. Since the configurations of particle packing is chosen on a random basis, which significantly complicated the calculations, the longitudinal and transverse diameters of particle clusters, the distance between centers of two largest particles, and similar natural geometric properties have been considered as characteristic system dimensions. Particle sizes, particle substance matter and some other values have been varied.

We assume that heat transfer in such a system is quasi-stationary and ruminate on sequential optimization following on from the necessity to minimize the next functional:

$$
\int_{\Omega+\Gamma}\left|T(r)-T_{3}(r)\right|^{2} d r \rightarrow \min ,
$$

where $T_{3}(r)$ is predetermined temperature. Other options for minimizing a functional are presented in [14, 15]. If surfaces of all particles have the same temperature, then the abovementioned expression is converted to: 


$$
\left|T\left(S_{i}\right)-T_{3}\right| \rightarrow \min
$$

An analytical solution has been obtained for the system "two spherical particles - continuum medium" $[10,11]$ to distribute temperature in a system of two particles in a bispherical coordinate system. Differentiating this solution, we have obtained equations for extremum parameters of the problem: $q_{1}^{*}, q_{2}^{*}$, $a_{1}^{*}, a_{2}^{*}$ where $q_{i}^{*}$ are the average particle densities of heat sources and $a_{i}^{*}$ are particle radii. That corresponds to the solution of the optimization problem (4). Solution for the case of two spherical particles, where the source intensity $I$ and the distance between two centers of spheres $d$ are chosen as control parameters, has been obtained in $[10,11]$.

One can determine the optimal parameters by solving a system of equations.

$$
\frac{\partial T_{i}}{\partial q_{i}}=0, \quad T_{i}=T_{3}, \frac{\partial T_{i}}{\partial a_{i}}=0, \quad T_{i}=T_{3}
$$

In numerical model calculations the authors assume that $\xi=\xi_{i}$ (on the particle surface). To reduce the number of parameters, the following quantities have been introduced:

$$
k=\frac{d}{a_{1}}, m=\frac{a_{2}}{a_{1}}, p=\frac{q_{2}}{q_{1}}, v=\frac{\aleph_{1}}{\aleph_{e}}
$$

Theparametersvarywithin:

$$
k=2 \div 500, m=0.01 \div 100
$$

It has been found that the optimal values of typical particle size relations depend on the substance of particles, their optical properties and the geometry of a system. Representative diagrams of such dependencies are shown in Fig. 4.

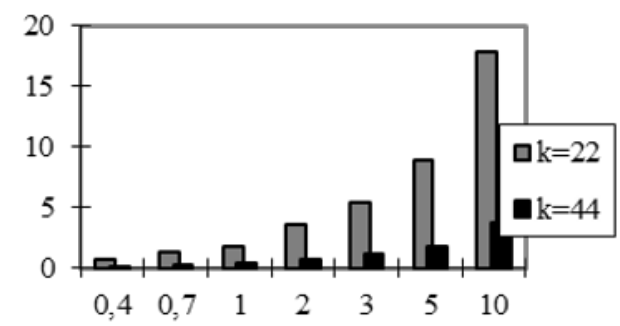

Fig. 4. Optimal dependence of particle radius on heat source relation, with $\eta=\pi / 3, m=10, v=5, \mathrm{k}=22$; 44

Based on the calculation of source relation for the water-air system, it follows that the dependence of the source on coordinate $\eta$ is nonmonotonic.

In recent years, the intensive investigations have been carried out on such structures as clusters by methods of computational physics. They are directly related to the processes of solid particles association that is cluster formation in clouds, coagulation of particles in fumes, formation of structures under relaxation of metal vapor, etc. If an aerosol particle $10-100 \mu \mathrm{m}$ in size is affected by laser radiation of a sufficiently high power, the particle breaks up into several particles (a cluster). If the internal energy of such "fragments" is close to the internal energy of systems governed by equations of a continuous medium, the proposed theory can be used to describe heat transfer in systems including clusters.

\section{Conclusion}

1. It follows from the calculations for a system of two spherical particles that the average value of heat source density essentially depends on the location of a twoparticle system relative to the wave vector and the electric field vector. It is clear that the influence of the second particle on the heat sources density distribution in the interior of the first one is great, even a distance between particles is large. Not only electromagnetic, but thermal interaction of dispersed particles can be much in evidence at large distances between their centers. The calculations suggest that near the boundary of a dispersed particle the temperature distribution is more inhomogeneous than in the central regions. This brings us to a conclusion that there exists a thermal surface layer of the particle, which is characterized by a strongly inhomogeneous temperature field inside. When studying the thermal interaction of two dispersed particles with significantly different absorbing properties, the authors have found that there occurs a thermal destruction of a low-absorbing particle caused by a temperature jump due to electromagnetic resonance in the adjacent highabsorbing particle.

2. The computational experiments for two-layer cylinders with concentric and non-concentric layers of different chemical composition with a randomly selected internal boundary between layers have shown that the density distributions of internal heat sources (proportional to a square of the absorbed field electric vector amplitude), depending on the angle in a polar coordinate system, take a form on the selected crosssection dependent on the outer layer thickness.

3. To study heat transfer in dispersed systems of different composition, configuration, and particle sizes, computational experiments have been carried out. The outcome of a finite element method analysis is therefore that the presence of particles of different sizes and thermophysical properties leads to an increase in temperature field nonuniformity in a system, with nonlinear effects being observed under a linear source dependence on temperature, while a nonlinear dependence amplifies these effects. If a system is polydisperse, the presence of larger particles has a significant impact on the distribution, and under boundary conditions of the third kind at the external system boundary collective effects can change the temperature one-and-a-half times or even more. This influence is the greater, the more heterogeneous the system (in particle size and composition).

This work is supported by the Russian Scientific Foundation (grant No. 18-11-00247). We thank Russian Science Foundation (grant №18-71-00137) and the Russian Ministry of Science and Education (grants 1.6198.2017/6.7 and 
1.7706.2017/8.9) for support. We also thank the Center of Collective Use of MSUT "STANKIN" for providing resources.

\section{References}

1. A.A. Lushnikov, A.A. Zagainov, I.E. Agranovsky, Yu.S. Lyubovtseva, Journal of Physical Chemistry 82(10), 1950-1958 (2008)

2. A.A. Lushnikov, A.A. Zagainov, Yu.S. Lyubovtseva, Chemical Physics, 34(10), 51 (2015)

3. F.G. Mitri, Journal of Quantitative and Radiative Transfer, 206, 142-150 (2018)

4. F.G. Mitri, Annals of Physics, 386, 1-14 (2017)

5. A.V. Barakov, A.P. Byrdin, A.A. Nadeev, Scientific Herald of the Voronezh State University of Architecture And Civil Engineering. Construction And Architecture, 3(31), 18-26 (VSACU, Voronezh, 2016)

6. A.P. Prishivalko, Optical and thermal fields inside light-scattering particles (NaukaiTekhnika, Minsk, 1983)

7. M. Bourne, E. Wolf, Fundamentals of Optics (Nauka, Moscow, 1970).

8. N.Ya. Vilenkin, Special functions and group representation theory (Nauka, Moscow, 1956).

9. N.I. Gamayunov, I.V. Krivenko., L.A. Uvarova, Yu.Z. Bondarev, ZhFKh.71(2), 2270-2274 (1997)

10. L.A. Uvarova, I.V. Krivenko., M.A. Smirnova, A.F. Ivannikov, Nonlinearity: Problems, Solutions and Applications, 519-541 (NY: Nova Science Publishers, Inc., 2017)

11. L.A. Uvarova, I.V. Krivenko., M.A. Smirnova, A.F. Ivannikov, Small absorbing particles in electromagnetic field and heat transfer in dispersed systems: Monograph,192 (Janus-K, Moscow, 2014)

12. G. Korn, Handbook on mathematics for scientists and engineers (Nauka, Moscow, 1984)

13. F.M. Morse, G. Feshbach, Methods of theoretical physics (Izd-voInostr. Literatury, Moscow1958)

14. M.A. Smirnova, Fundamental Physical and Mathematical Problems and Simulation of Technical and Technological Systems 3, 87-95 ("Stankin”, Moscow, 2000)

15. M.A. Smirnova, Fundamental Physical and Mathematical Problems and Simulation of Technical and Technological Systems 6, 212-226 ("Stankin”, Moscow, 2003) 